\title{
RESULTS ON COMMON FIXED POINTS ON COMPLETE METRIC SPACES
}

\author{
by BRIAN FISHER
}

(Received 26 April, 1979)

The following theorem was proved in [1].

THEOREM 1. Let $S$ and $T$ be continuous, commuting mappings of a complete, bounded metric space $(X, d)$ into itself satisfying the inequality

$$
\begin{gathered}
d\left(S^{\mathrm{p}} T^{\mathrm{p}^{\prime}} x, S^{\mathrm{a}} T^{\mathrm{q}^{\prime}} y\right) \leqslant c \cdot \max \left\{d\left(S r T^{\prime^{\prime}} x, S^{s} T^{s^{\prime}} y\right), d\left(S^{r} T^{r^{\prime}} x, S^{\rho} T^{\rho^{\prime}} x\right), d\left(S^{s} T^{s^{\prime}} y, S^{\sigma} T^{\sigma^{\prime}} y\right):\right. \\
\left.0 \leqslant r, \rho \leqslant p ; 0 \leqslant r^{\prime}, \rho^{\prime} \leqslant p^{\prime} ; 0 \leqslant s, \sigma \leqslant q ; 0 \leqslant s^{\prime}, \sigma^{\prime} \leqslant q^{\prime}\right\}
\end{gathered}
$$

for all $x, y$ in $X$, where $0 \leqslant c<1$ and $p, p^{\prime}, q, q^{\prime} \geqslant 0$ are fixed integers with $p+p^{\prime}, q+q^{\prime} \geqslant 1$. Then $S$ and $T$ have a unique common fixed point $z$. Further, if $p^{\prime}$ or $q^{\prime}=0$, then $z$ is the unique fixed point of $S$ and if $p$ or $q=0$, then $z$ is the unique fixed point of $T$.

It was shown that the condition that $S$ and $T$ commute was necessary in this theorem. It is possible however that the condition that $X$ be bounded is not necessary in this theorem. We now prove the following theorem which does not require $S$ and $T$ to commute or $X$ to be bounded.

THeOrem 2. Let $S$ and $T$ be continuous mappings of a complete metric space $(X, d)$ into itself satisfying the inequality

$$
d\left(S^{p} x, T^{q} y\right) \leqslant c . \max \left\{d\left(S^{r} x, T^{s} y\right): 0 \leqslant r \leqslant p ; 0 \leqslant s \leqslant q\right\}
$$

for all $x, y$ in $X$, where $0 \leqslant c<1$ and $p, q$ are fixed positive integers. Then $S$ and $T$ have a unique common fixed point $z$. Further, $z$ is the unique fixed point of $S$ and $T$.

Proof. Let $x$ be an arbitrary point in $X$ and put

$$
A=\max \left\{d\left(T^{s} x, T^{q} x\right): 0 \leqslant s \leqslant q\right\} .
$$

Suppose that the sequence $\left\{S^{n} x: n=1,2, \ldots\right\}$ is unbounded. Then there exists an integer $n \geqslant p$ such that

$$
d=d\left(S^{n} x, T^{q} x\right) \geqslant \max \left\{d\left(S^{r} x, T^{q} x\right): 0 \leqslant r \leqslant n\right\}
$$

with

$$
d>c A /(1-c) .
$$

Thus

$$
d\left(S^{r} x, T^{s} x\right) \leqslant d\left(S^{r} x, T^{a} x\right)+d\left(T^{q} x, T^{s} x\right) \leqslant d+A
$$

for $0 \leqslant r \leqslant n$ and $0 \leqslant s \leqslant q$. On using inequality (1), it now follows that

$$
d=d\left(S^{n} x, T^{q} x\right) \leqslant c, \max \left\{d\left(S^{r} x, T^{s} x\right): n-p \leqslant r \leqslant n ; 0 \leqslant s \leqslant q\right\} \leqslant c(d+A)
$$

Glasgow Math. J. 21 (1980) 165-167. 
and so $d \leqslant c A /(1-c)$ giving a contradiction. This contradiction implies that the sequence $\left\{S^{n} x: n=1,2, \ldots\right\}$ must be bounded.

Similarly, we can prove that the sequence $\left\{T^{n} x: n=1,2, \ldots\right\}$ is bounded and so

$$
M=\sup \left\{d\left(S^{r} x, T^{s} x\right): r, s=0,1,2, \ldots\right\}
$$

is finite. Now for arbitrary $\varepsilon>0$, choose a positive integer $N$ such that $c^{N} M<\varepsilon$. It follows that for $m, n \geqslant N \cdot \max \{p, q\}$

$$
\begin{aligned}
d\left(S^{m} x, T^{n} x\right) & \leqslant c \cdot \max \left\{d\left(S^{r} x, T^{s} x\right): m-p \leqslant r \leqslant m ; n-q \leqslant s \leqslant n\right\} \\
& \leqslant c^{2} \cdot \max \left\{d\left(S^{r} x, T^{s} x\right): m-2 p \leqslant r \leqslant m ; n-2 q \leqslant s \leqslant n\right\} \\
& \leqslant c^{N} \cdot \max \left\{d\left(S^{r} x, T^{s} x\right): m-N p \leqslant r \leqslant m ; n-N q \leqslant s \leqslant n\right\} \\
& \leqslant c^{N} M<\varepsilon
\end{aligned}
$$

and so

$$
d\left(S^{m} x, S^{r} x\right) \leqslant d\left(S^{m} x, T^{n} x\right)+d\left(T^{n} x, S^{r} x\right)<2 \varepsilon
$$

for $m, n, r \geqslant N . \max \{p, q\}$. Thus $\left\{S^{n} x: n=1,2, \ldots\right\}$ is a Cauchy sequence in the complete metric space $X$ and so has a limit $z$ in $X$. Further, since

$$
d\left(S^{n} x, T^{n} x\right)<\varepsilon
$$

for $n \geqslant N . \max \{p, q\}$. the sequence $\left\{T^{n} x: n=1,2, \ldots\right\}$ also converges to $z$. From the continuity of $S$ and $T$ it now follows immediately that $z$ is a common fixed point of $S$ and $T$.

Now suppose that $w$ is a second fixed point of $T$. Then

$$
\begin{aligned}
d(z, w) & =d\left(S^{p} z, T^{q} w\right) \\
& \leqslant c \cdot \max \left\{d\left(S^{r} z, T^{s} w\right): 0 \leqslant r \leqslant p ; 0 \leqslant s \leqslant q\right\} \\
& =c d(z, w)
\end{aligned}
$$

proving that $z=w$, since $c<1$. Similarly we can prove that $z$ is the unique fixed point of $S$. This completes the proof of the theorem.

Corollary 1. Let $S$ be a mapping and let $T$ be a continuous mapping of a complete metric space $(X, d)$ into itself satisfying the inequality

$$
d\left(S x, T^{q} y\right) \leqslant c . \max \left\{d\left(S^{r} x, T^{s} y\right): 0 \leqslant r \leqslant 1 ; 0 \leqslant s \leqslant q\right\}
$$

for all, $x, y$ in $X$, where $0 \leqslant c<1$ and $q$ is a fixed positive integer. Then $S$ and $T$ have a unique common fixed point $z$. Further, $z$ is the unique fixed point of $S$ and $T$.

Proof. Let $x$ be an arbitrary point in $X$. Then as in the proof of Theorem 2, the sequences $\left\{S^{n} x: n=1,2, \ldots\right\}$ and $\left\{T^{n} x: n=1,2, \ldots\right\}$ convęrge to a point $z$ in $X$. Since $T$ 
is continuous, $z$ is a fixed point of $T$. Further

$$
\begin{aligned}
d(S z, z) & =d\left(S z, T^{q} z\right) \\
& \leqslant c \cdot \max \left\{d\left(S^{r} z, T^{s} z\right): 0 \leqslant r \leqslant 1 ; 0 \leqslant s \leqslant q\right\} \\
& =c d(S z, z)
\end{aligned}
$$

proving that $S z=z$, since $c<1$. Thus $z$ is a common fixed point of $S$ and $T$. The uniqueness of $z$ follows from the proof of the theorem, since the continuity of $S$ was not used to prove the uniqueness.

Corollary 2. Let $S$ and $T$ be mappings of a complete metric space $(X, d)$ into itself satisfying the inequality

$$
d(S x, T y) \leqslant c \cdot \max \{d(x, y), d(x, T y), d(y, S x)\}
$$

for all $x, y$ in $X$, where $0 \leqslant c<1$. Then $S$ and $T$ have a unique common fixed point $z$. Further, $z$ is the unique fixed point of $S$ and $T$.

Proof. Let $x$ be arbitrary point in $X$. Then again the sequences $\left\{S^{n} x: n=1,2, \ldots\right\}$ and $\left\{T^{n} x: n=1,2, \ldots\right\}$ converge to a point $z$ in $X$. Further

$$
\begin{aligned}
d(S z, z) & \leqslant d\left(S z, T^{n} x\right)+d\left(T^{n} x, z\right) \\
& \leqslant c \cdot \max \left\{d\left(z, T^{n-1} x\right), d\left(z, T^{n} x\right),\left(T^{n-1} x, S z\right)\right\}+d\left(T^{n} x, z\right) .
\end{aligned}
$$

Letting $n$ tend to infinity it follows that

$$
d(S z, z) \leqslant c d(S z, z)
$$

proving that $S z=z$, since $c<1$. Similarly, we can prove that $z$ is also a fixed point of $T$. The uniqueness of $z$ again follows from the proof of the theorem.

AckNowledgement. The author would like to thank the referee for his helpful suggestions towards the improvement of this paper.

\section{REFERENCE}

1. B. Fisher, Results on common fixed points on bounded metric spaces, Math. Sem. Notes Kobe Univ., 7 (1979), 73-80.

\section{Department of Mathematics}

THE UNIVERSITY

LEICESTER LE1 7RH 